\title{
Frizzled 2 mediates the migration and invasion of human oral squamous cell carcinoma cells through the regulation of the signal transducer and activator of transcription-3 signaling pathway
}

\author{
ENJIAO ZHANG, ZHENNING LI, ZHONGFEI XU, WEIYI DUAN, CHANGFU SUN and LI LU \\ Department of Oral and Maxillofacial Surgery, Department of Oromaxillofacial-Head and Neck Surgery, \\ School of Stomatology, China Medical University, Shenyang, Liaoning 110002, P.R. China
}

Received June 4, 2015; Accepted July 1, 2015

DOI: $10.3892 /$ or.2015.4285

\begin{abstract}
Frizzled2 (Fzd2) is a receptor for wingless-type MMTV integration site family members (Wnts), the aberrant overexpression of which has been noted to contribute to cancer metastasis. The present study was performed to characterize the role of $\mathrm{Fzd} 2$ in the migration and invasion of oral squamous cell carcinomas (OSCC) in vitro. Using TSCCa cells (a tongue SCC cell line) for loss- or gain-of-function of Fzd2, we found that a forced overexpression of $\mathrm{Fzd} 2$ promoted TSCCa cell migration and invasion, decreased the expression of epithelial-cadherin (E-cadherin, an epithelial marker) and increased that of vimentin, Snail Slug, matrix metalloproteinases (MMPs)-2/-9/-13 and a-disintegrin and metalloproteinase with thrombospondin motifs-5 (ADAMTS5). By contrast, RNA interference (RNAi)-mediated knockdown of Fzd2 had opposite effects on OSCC cells. In addition, we found that the phosphorylation of signal transducer and activator of transcription-3 (STAT3) was enhanced by Fzd2 overexpression, but suppressed by Fzd2 depletion, and that STAT3-specific shRNA attenuated Fzd2 overexpression-induced cell invasion. In summary, the present study demonstrated that Fzd2 contributes to the migration and invasion of OSCC cells, at least partly through regulation of the STAT3 pathway. These results suggest Fzd2 as a novel therapeutic target for OSCC.
\end{abstract}

\section{Introduction}

Oral squamous cell carcinoma (OSCC) commonly develops on the surface of the tongue (1), larynx (2) or lip (3), the carcinogenesis of which involves multistep malignant transformation

Correspondence to: Dr Li Lu, Department of Oral and Maxillofacial Surgery, Department of Oromaxillofacial-Head and Neck Surgery, School of Stomatology, China Medical University, 117 North Nanjing Street, Shenyang, Liaoning 110002, P.R. China E-mail: cmululi@163.com

Key words: oral squamous cell carcinomas, Frizzled2, cell migration, cell invasion, signal transducer and activator of transcription-3 signaling pathway of the surface squamous epithelium (4). In accordance with previous studies (5), an earlier epidemiological analysis for patients with oral cancers in China has shown that over a half of the examined oral malignancies are SCC (6), suggesting SCC as the predominate oral malignancy. Despite recent advances in novel therapies (7), the prognosis for patients with OSCC remains poor (8). Thus, it is imperative to identify a novel therapeutic target in OSCC.

Frizzled2 (Fzd2) is a wingless-type MMTV integration site family member (Wnt) receptor that was first cloned and characterized by Sagara et al (9). As is the case for other Fzd isoforms, Fzd2 has seven transmembrane domains, a cysteine-rich domain in the $\mathrm{N}$-terminal extracellular region and a C-terminal Ser/Thr-Xxx-Val motif (9). Fzds and their ligands Wnts were initially observed to play important roles in regulating embryonic development (10). Inappropriate activation of the Wnt/Fzd pathway has been reported in numerous types of human cancer (11), such as hepatocellular carcinoma (12) and colorectal cancer (13). Targeting of frizzled receptors decreases the growth and tumorigenicity of human pancreatic, breast and non-small cell lung tumors (14). Despite these previous studies suggesting an oncogenic property of Fzd2, Ding et al contrarily identified Fzd2 as a tumor suppressor in salivary adenoid cystic carcinomas in vitro (15). Since Fzd2 is frequently overexpressed in head and neck SCC cancer cell lines as compared with normal bronchial epithelial cells or primary oral squamous epithelial cells (16), it is likely that the aberrant overexpression of Fzd2 contributes to OSCC carcinogenesis. Notably, Prgomet et al have found that the ectopic expression of Wnt5a, a ligand for Fzd2, promotes OSCC cell migration and invasion (17). However their study did not elucidate whether Fzd2 also participated in OSCC aggressiveness.

Therefore, the present study was conducted to investigate whether and how Fzd2 affected OSCC cell migration and invasion in a tongue SCC cell line and TSCCa cells. Stable overexpression and knockdown of Fzd 2 were performed in TSCCa cells. In addition, the underlying mechanisms were examined.

\section{Materials and methods}

Cell culture. Human tongue SCC cancer cells (TSCCa cells) were purchased from the Cell Bank of Wuhan University 
(Wuhan, China) and cultured in Dulbecco's modified Eagle's medium (DMEM; Gibco, Grand Island, NY, USA) supplemented with $10 \%$ fetal bovine serum (FBS; HyClone, Logan, UT, USA), $100 \mathrm{U} / \mathrm{ml}$ penicillin and $100 \mu \mathrm{g} / \mathrm{ml}$ streptomycin at $37^{\circ} \mathrm{C}$ in a humidified atmosphere of $5 \% \mathrm{CO}_{2}$.

Plasmid construction, stable and transient transfection. Overexpression of Fzd2 in TSCCa cells was performed using pcDNA3.1+ vector (Clontech Laboratories, Inc., Mountain View, CA, USA). Briefly, a pair of primers was designed as forward, 5'-ctacaagcttagcatgcggcccegcag-3' and reverse, 5'-aatctcgagcgtccetcacacggtggtctca-3' and synthesized to amplify the complete coding sequence (CDS) area of $F z d 2$ gene (NM_001466.3) via reverse transcription PCR (RT-PCR). The obtained fragments were first subcloned into the UltraPower pUM-T simple vector (BioTeke, Beijing, China) between HindIII and XhoI restriction enzyme, sites sequenced, and then inserted into the pcDNA3.1+ vector (pcDNA3.1-Fzd2). The empty pcDNA3.1+ vector served as a control (pcDNA3.1-NC). For knockdown of Fzd2 in cancer cells, pRNA-H1.1 vector (GenScript Biotechnology Co., Ltd., Nanjing, China) was utilized to generate shRNA plasmids targeting Fzd2 mRNA in the present study. Briefly, a pair of oligonucleotides encoding shRNA targeting Fzd2 mRNA (target sequence, 5'-gaggccaactctcagtact-3'; 1,341-1,359 bp) was synthesized, annealed and inserted into the pRNA-H1.1 vector (pRNA-H1.1-Fzd2 shRNA). Scrambled shRNA was also obtained and inserted into the pRNA-H1.1 plasmid as the negative control (pRNA-H1.1-NC).

For establishment of oral cancer cells with increased or decreased Fzd 2 expression, G418 (Invitrogen Life Technologies, Carlsbad, CA, USA) screen was performed. TSCCa cells $\left(3 \times 10^{5}\right)$ were first seeded in 6-well plates and allowed to grow for $24 \mathrm{~h}$. The cells were transfected with pcDNA3.1Fzd2, pRNA-H1.1-Fzd2 shRNA or negative control plasmids (2 $\mu \mathrm{g}$ for each) using Lipofectamine 2000 (Invitrogen Life Technologies) according to the manufacturer's intstructions. At $24 \mathrm{~h}$-post transfection, G418 $(200 \mu \mathrm{g} / \mathrm{ml})$ was added into the cell culture to screen the cells for 1-2 weeks. Cell clones that resisted G418 were selected and assayed for Fzd2 mRNA and protein expression.

To examine whether the signal transducer and activator of transcription-3 (STAT3) signaling pathway was involved in Fzd2-mediated TSCCa cell metastasis, shRNA targeting STAT3 mRNA (NM_139276.2; target sequence, 5'-ggtgtctccactggtctat-3'; 2,238-2,256 bp) was obtained and used to transiently transfect TSCCa cells using Lipofectamine 2000. After $24 \mathrm{~h}$ the cells transfected with pRNA-H1.1-STAT3 shRNA plasmid were subjected to further analysis.

Quantitative PCR. Total RNAs were isolated from cell lysates using an RNA Simple Total RNA kit (Tiangen, Beijing, China), and then processed for cDNA synthesis with a Super M-MLV Reverse Transcriptase kit (BioTeke Corp., Beijing, China). Fzd2 mRNA levels were determined using SYBRGreen (Solarbio, Beijing, China), and normalized to $\beta$-actin via a comparative threshold cycle (CT) method $\left(2^{-\Delta \Delta C t}\right)(18)$. Primers used for determining Fzd2 mRNA expression were as follows: forward, 5'-cacggacatcgectacaacc-3' and reverse, 5 '-gcaccttcaccagcggatag-3'.
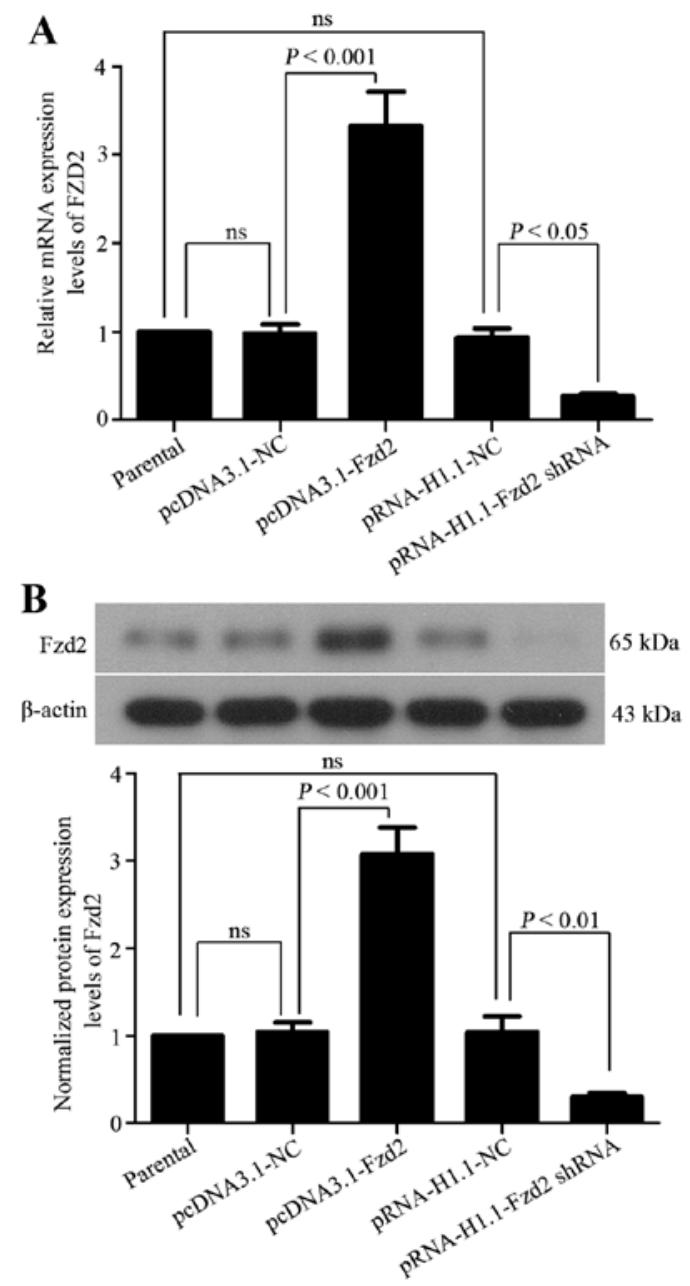

Figure 1. Detection of Fzd2 mRNA and protein expression levels in TSCCa cells. (A) Fzd 2 mRNA expression levels were determined by quantitative PCR. (B) Representative protein bands of Fzd 2 were determined by western blot analysis, and the band density values were calculated as a ratio to $\beta$-actin. Data are presented as the mean \pm standard deviation $(\mathrm{SD})(\mathrm{n}=3) .{ }^{\mathrm{n} s} \mathrm{P}>0.05$ Fzd2, frizzled2.

Western blot analysis. Total proteins were extracted from cells with NP-40 lysis buffer (Beyotime, Shanghai, China), fractionated on sodium dodecyl sulphate-polyacrylamide gel electrophoresis (SDS-PAGE), transferred onto polyvinylidene fluoride (PVDF) membranes (Millipore, Bedford, MA, USA) and blocked with $5 \%(\mathrm{w} / \mathrm{v})$ skim milk. The membranes were incubated with goat polyclonal antibodies against Fzd2 (1:200 dilution) and phosphorylated p-STAT3 ${ }^{\text {Tyr }} 705$ (1:200 diluted) (both from Santa Cruz Biotechnology, Inc., Santa Cruz, CA, USA), and rabbit polyclonal antibodies against epithelialcadherin (E-cadherin; 1:400 dilution; Boster, Wuhan, China), vimentin (1:500 dilution), Snail (1:500 dilution), Slug (1:500 dilution) (all from Bioss, Beijing, China), matrix metalloproteinase (MMP)-2 (1:400 dilution), MMP-9 (1:400 dilution) (both from Boster), MMP-13 (1:500 dilution; Bioss), a-disintegrin and metalloproteinase with thrombospondin motifs-5 (ADAMTS5; 1:250 dilution; Abcam, Cambridge, UK) and total STAT-3 (1:200 dilution; Santa Cruz Biotechnology, Inc.) at $4^{\circ} \mathrm{C}$ overnight. HRP-conjugated secondary antibodies were used to incubate the membranes for $45 \mathrm{~min}$ at $37^{\circ} \mathrm{C}$. The protein blots were visualized with an enhanced chemiluminescence 


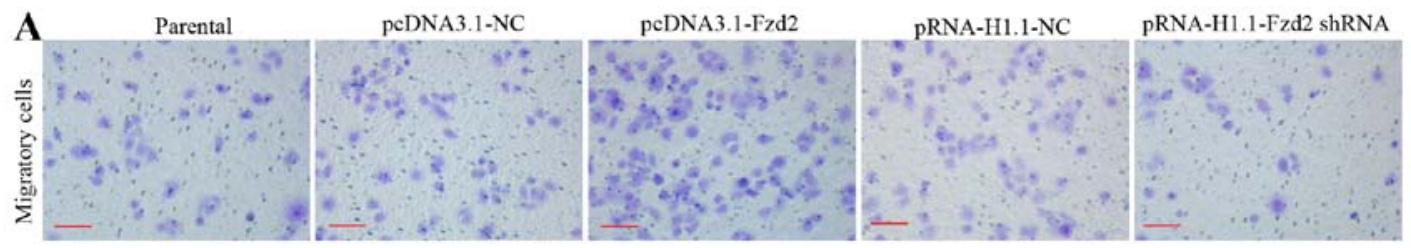

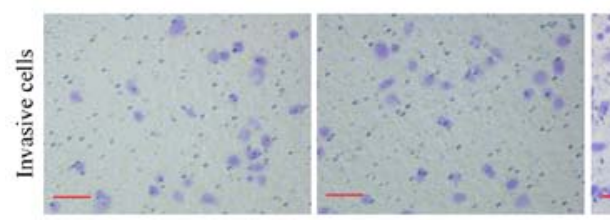

B

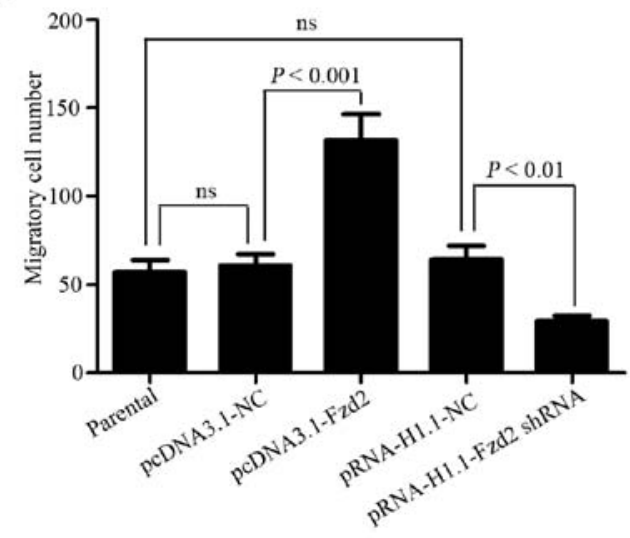

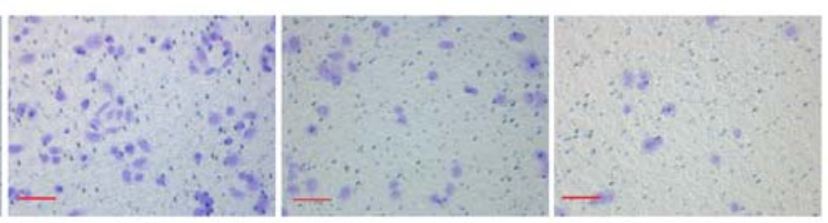

C

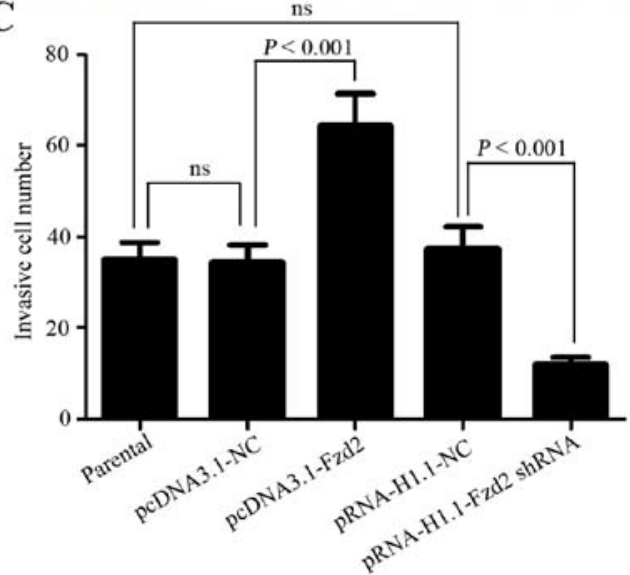

Figure 2. Fzd2 mediates the migration and invasion of TSCCa cells. (A) TSCCa cell migration was detected by Matrigel-free Transwell inserts, while the cell invasion was determined by Matrigel-coated Transwell inserts. (B) Migratory and (C) invasive cells were counted in five random areas of triplicate wells (scale bars, $100 \mu \mathrm{m}$ ). Data are presented as the mean \pm standard deviation $(\mathrm{SD}) .{ }^{\mathrm{n}} \mathrm{P}>0.05$. Fzd2, frizzled2.

(ECL) kit (Seven Seas Pharmtech Co., Ltd., Shanghai, China), and normalized to a protein density of endogenous $\beta$-actin.

Assessment of cell migration and invasion. Transwell inserts (8- $\mu \mathrm{m}$ pores; Corning Incorporated Life Sciences, Tewksbury, MA, USA) coated with or without Matrigel (BD Biosciences, San Jose, CA, USA) were used to detect cell invasion and migration, respectively. For the invasion assay, the upper surface of the filter was first coated with Matrigel. Cancer cells $\left(2 \times 10^{4}\right)$ in $200 \mu 1$ serum-free DMEM medium were placed onto the upper chambers, and the lower chambers were filled with complete media. After $24 \mathrm{~h}$, the inner surface of the upper chambers was wiped with cotton swabs, and the invasive cells were fixed with paraformaldehyde (Sinopharm, Shanghai, China) for $20 \mathrm{~min}$, and then stained with hematoxylin for $5 \mathrm{~min}$. The stained cells were then counted in five random areas in triplicate wells. A cell migration assay was performed using the Transwell inserts without Matrigel.

Immunofluorescent staining. TSCCa cells were first grown to subconfluence on coverslips, fixed in paraformaldehyde and then permeabilized with $0.1 \%$ Triton X-100 (Amresco China, Shanghai, China). E-cadherin protein was immunodetected with a rabbit polyclonal antibody against E-cadherin (1:200 dilution; Boster) and Cy3-conjugated secondary antibody, and the nuclei were visualized with 4',6-diamidino-2-phenylindole (DAPI; Biosharp, Hefei, China). Thereafter, the coverslips were mounted onto glass slides and viewed under a microscope (Olympus, Tokyo, Japan).
Statistical analysis. Data are presented as the mean \pm standard deviation (SD), and were assessed by one-way analysis of variance followed by the Bonferroni post hoc test. $\mathrm{P}<0.05$ was considered to indicate a statistically significant result.

\section{Results}

Stable overexpression and silencing of Fzd2 in OSCC cells. To up- or downregulate Fzd2 expression in TSCCa cells that express a moderate level of Fzd2, pcDNA3.1-Fzd2, pRNAH1.1-Fzd2 shRNA plasmids or their corresponding negative control plasmids were constructed to transfect the TSCCa cells. The mRNA and protein expression levels of Fzd2 was then determined in cells resisting G418 with quantitative PCR and western blot analysis, respectively. The pcDNA3.1-Fzd2 plasmids led to a significant upregulation in Fzd2 expression in TSCCa cells (Fig. 1). In addition, Fzd 2 expression in the TSCCa cells transfected with the Fzd2 shRNA showed efficient depletion (Fig. 1). These results indicated that TSCCa cells with a higher or lower expression of Fzd2 were successfully established in the present study.

Fzd2 mediates OSCC cell migration and invasion in vitro. Transwell inserts coated with or without Matrigel were used to investigate the impact of Fzd2 on TSCCa cell invasion and migration, respectively. The results showed that the migratory cell number was increased from $60.8 \pm 6.3$ to $131.6 \pm 15$ when Fzd2 was overexpressed, but decreased from $64 \pm 8.2$ to 29.2 \pm 3.3 when Fzd2 was underexpressed (Fig. 2A and B). 

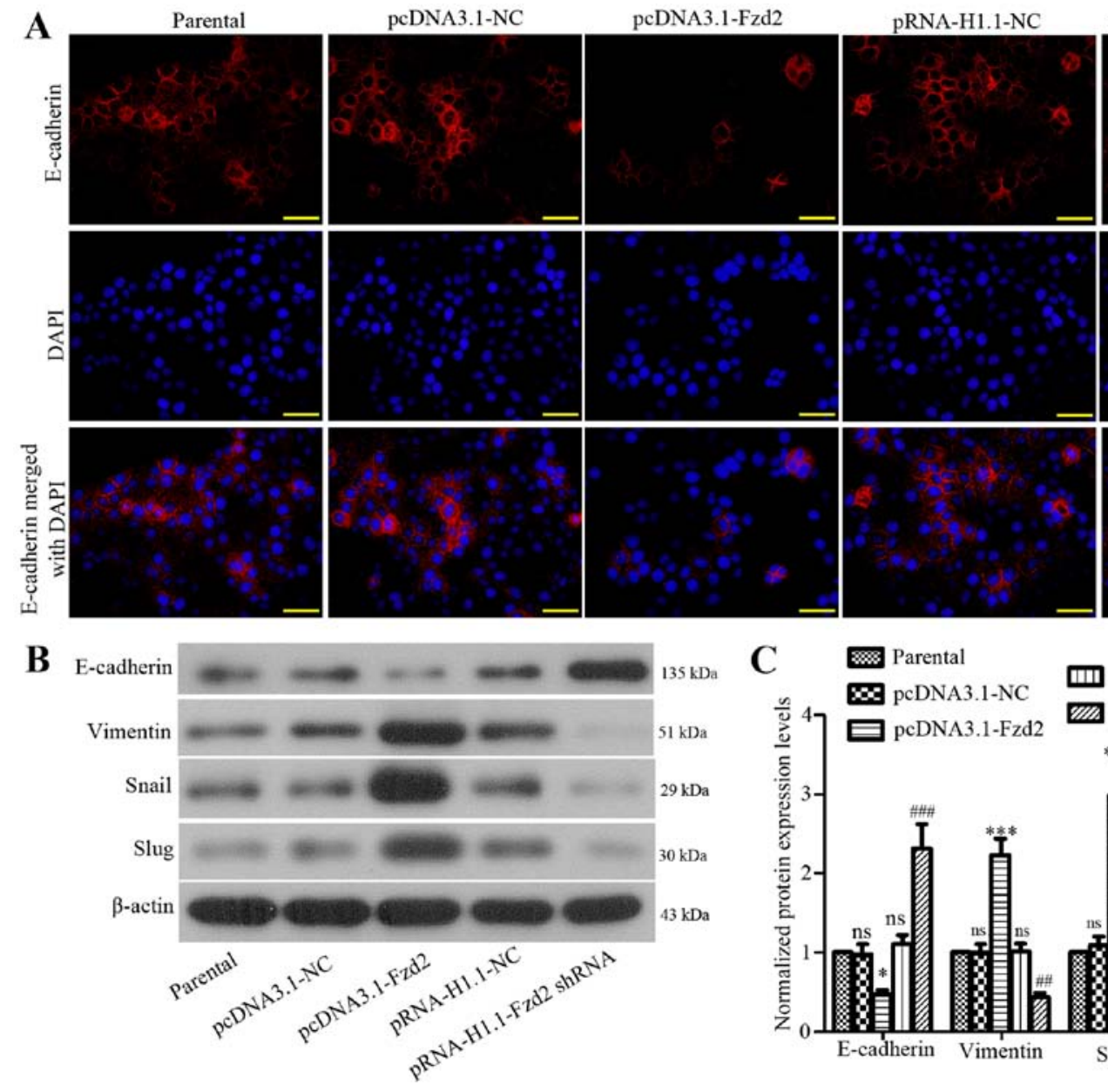

pRNA-H1.1-Fzd2 shRNA
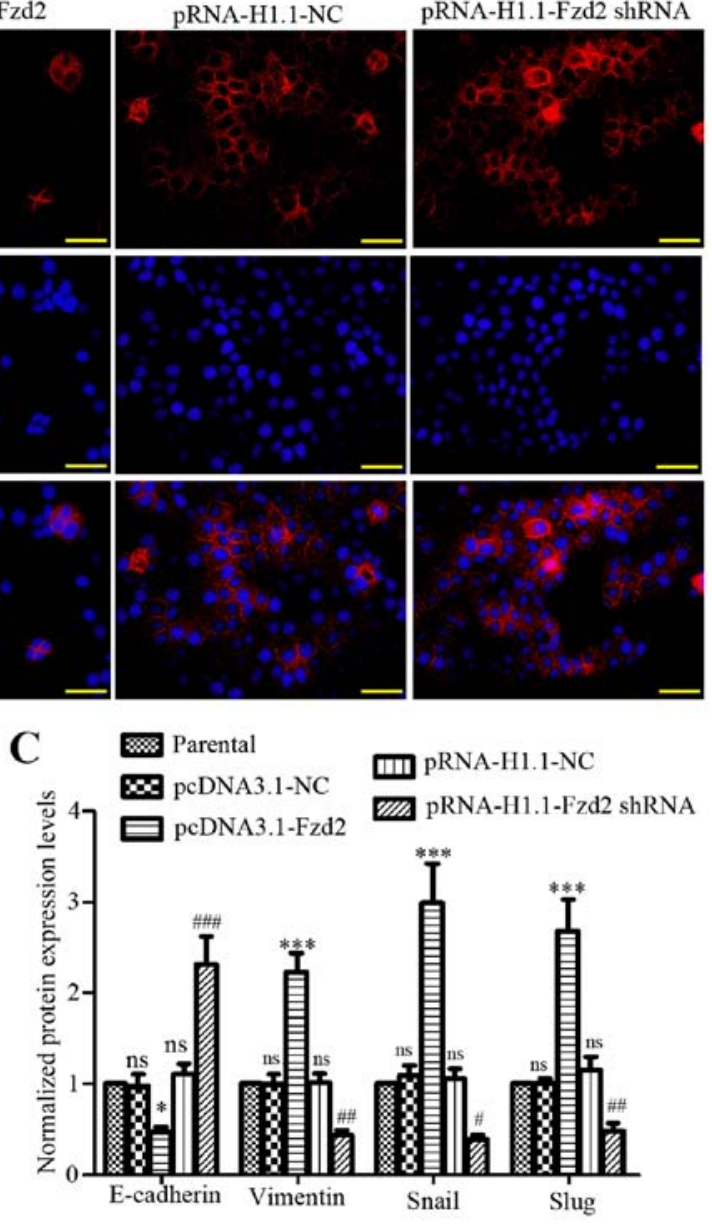

Figure 3. Fzd2 promotes EMT in TSCCa cells. (A) Representative images of immunofluorescent staining against E-cadherin in TSCCa cancer cells (scale bars, $50 \mu \mathrm{m}$ ). (B) Representatives of proteins bands of E-cadherin, vimentin, Snail and Slug in TSCCa cells determined by western blot analysis, and (C) normalized to the endogenous control $\beta$-actin. Data are presented as the mean \pm standard deviation $(\mathrm{SD})(\mathrm{n}=3)$. ${ }^{\mathrm{n}} \mathrm{P}>0.05$ vs. the parental group; ${ }^{*} \mathrm{P}<0.05$, ${ }^{* * * *} \mathrm{P}<0.001 \mathrm{vs}$. the pcDNA3.1-NC group; ${ }^{\# P}<0.05,{ }^{\#} \mathrm{P}<0.01,{ }^{\# \#} \mathrm{P}<0.001$ vs. the pRNA-H1.1-NC group. Fzd2, frizzled2; EMT, epithelial-mesenchymal transition.

Similar change patterns of the invasive cell number were obtained from the invasion assay (pcDNA3.1-NC vs. pcDNA3.1-Fzd2=34.4 \pm 3.9 vs. $64.6 \pm 6.9$; pRNA-H1.1-NC vs. pRNA-H1.1-Fzd2 shRNA=37.4 \pm 4.9 vs. $12 \pm 1.6$; Fig. $2 A$ and $C$ ). These results revealed that the migratory and invasive capabilities of TSCCa cells were positively correlated with Fzd2 expression levels, suggesting that Fzd2 mediated the aggressive metastasis of OSCC cells in vitro.

Fzd2 promotes the epithelial-mesenchymal transition (EMT) in OSCC cells. Tumor cells usually undergo partial or full EMT, and are converted to migratory and invasive cells (19). We here determined whether Fzd2 played a role in the EMT process of TSCCa cells. The expression of E-cadherin (an epithelial marker) was significantly increased in TSCCa cells when Fzd2 was inhibited, but decreased when Fzd2 was overexpressed as determined by immunofluorescent staining (Fig. 3A). The subsequent western blot analysis confirmed the immunofluorescent staining results of E-cadherin (Fig. 3B and C). Moreover, the expression levels of the E-cadherin suppressors Snail and Slug, and mesenchymal marker vimentin (20) were increased in TSCCa cells after Fzd2 overexpression, and decreased following Fzd2 depletion (Fig. 3B and C). The results showed that Fzd2 contributed to OSCC cell EMT by mediating the above mentioned EMT-related proteins.

Fzd2 mediates the expression of multiple matrix metalloproteinases in OSCC cells. Degradation of extracellular matrix (ECM) enabled the invasive behavior of tumor cells (21). The involvement of zinc metalloproteinases, including MMPs and ADAMTSs, which are representative families, have been identified in oral cancer development $(22,23)$. We therefore examined whether the expression levels of important MMPs in TSCCa cells were affected by Fzd 2 in the present study. As compared with the negative control cells, the expression levels of MMP-2, -9 and -13 and ADAMTS5 were upregulated in Fzd2-overexpressed TSSCa cells, but downregulated in Fzd2-depleted TSSCa cells (Fig. 4).

STAT3 signaling pathway is involved in Fzd2-mediated OSCC cell invasion. Since the aberrant activation of STAT3 signaling transduction is involved in the invasive behaviors of cancer cells (24), we investigated whether this pathway was involved in Fzd2-mediated OSCC cell invasion. The western blot analysis showed that the phosphorylation of STAT3 was enhanced 
A

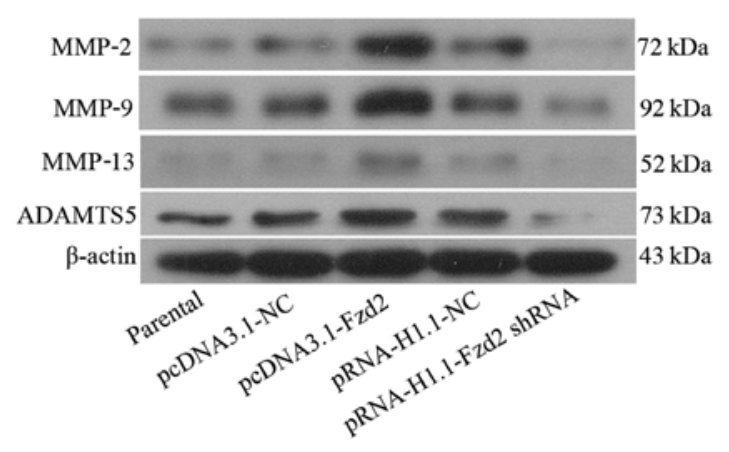

B

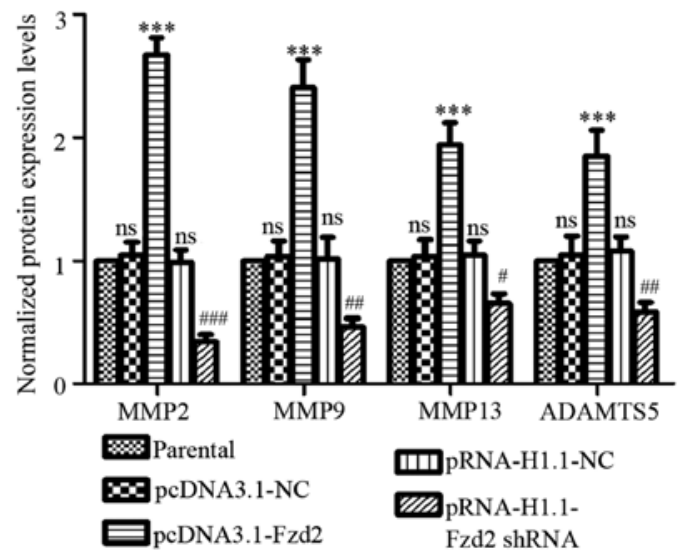

Figure 4. Effects of Fzd2 on matrix metalloproteases in TSCCa cells. (A) Representative images of proteins bands of MMP-2, -9 and -13 and ADAMTS5 in TSCCa cells were determined by western blot analysis, and (B) normalized to the endogenous control $\beta$-actin. Data are presented as the mean \pm standard deviation (SD) (n=3). ${ }^{\mathrm{ns}} \mathrm{P}>0.05$ vs. the parental group; ${ }^{* * *} \mathrm{P}<0.001$ vs. the pcDNA3.1-NC group; ${ }^{\#} \mathrm{P}<0.05,{ }^{\# \#} \mathrm{P}<0.01,{ }^{\# \# \#} \mathrm{P}<0.001$ vs. the pRNA-H1.1-NC group. Fzd2, frizzled2; MMP, matrix metalloproteinase; ADAMTS5, metalloproteinase with thrombospondin motifs-5.

A

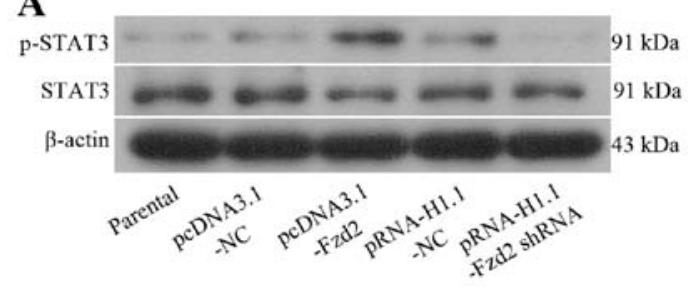

C

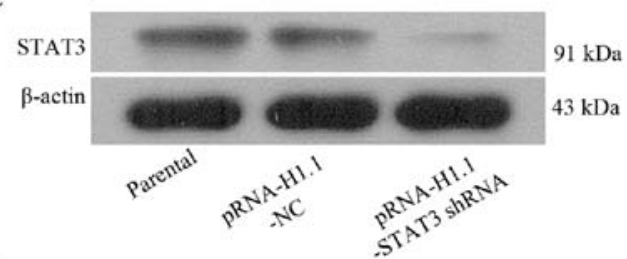

D
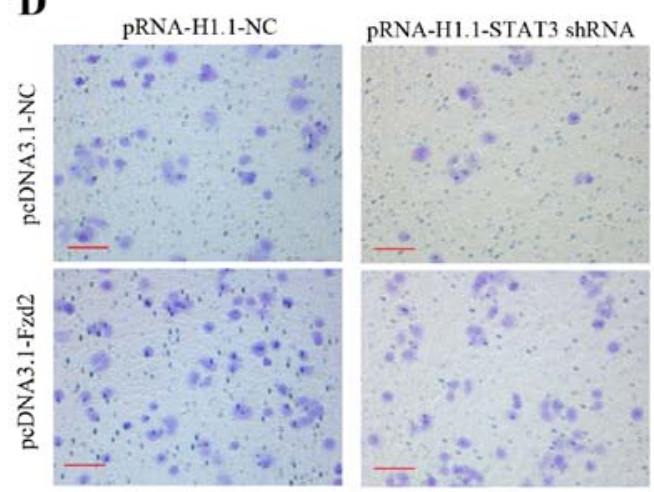

B

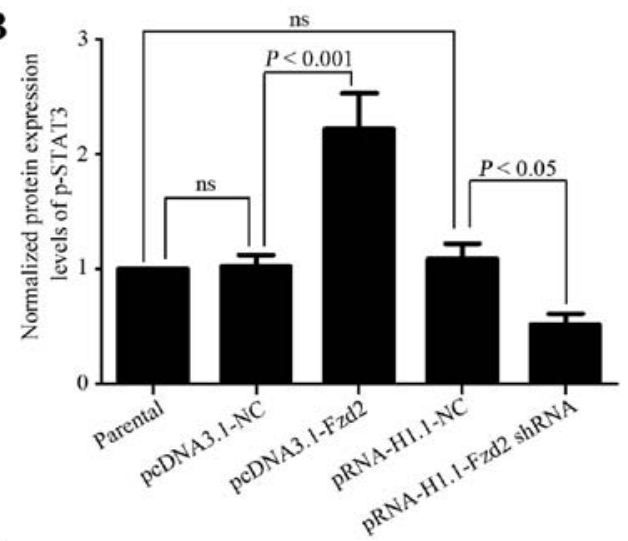

$\mathbf{E}$

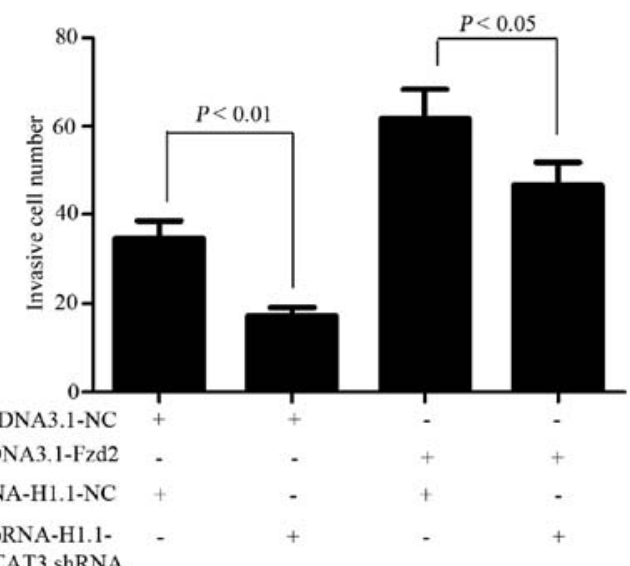

Figure 5. Fzd2 promotes TSCCa cell invasion partly through mediation of the STAT3 signaling pathway. (A and C) Representative images of proteins bands of total STAT3 and phospho-STAT3 in TSCCa cells determined by western blot analysis, and (B) normalized to the endogenous control $\beta$-actin. (D) Cell invasion was determined by Matrigel-coated Transwell inserts, and (E) the invasive cell numbers were counted in five random areas of triplicate wells (scale bars, $100 \mu \mathrm{m})$. Data are presented as the mean \pm standard deviation (SD) $(\mathrm{n}=3) .{ }^{\mathrm{n} s} \mathrm{P}>0.05$. Fzd2, frizzled2; STAT3, signal transducer and activator of transcription-3.

in TSCCa cells overexpressing Fzd2, but suppressed in those depleting Fzd2 (Fig. 5A and B). To examine whether the activated STAT3 signal contributed to Fzd2-induced OSCC cell invasion, a specific shRNA targeting STAT3 mRNA was used to transfect the TSCCa cells that overexpressed Fzd2. STAT3 expression in the TSCCa cells transfected with the STAT3
shRNA showed efficient depletion (Fig. 5C). The matrigel invasion assay revealed that the Fzd2 overexpression induced the invasion of TSCCa cells was attenuated when endogenous STAT3 was suppressed (Fig. 5D and E). These results suggested that Fzd 2 mediated OSCC cell invasion at least partly through mediation of the STAT3 signaling pathway. 


\section{Discussion}

Findings of a recent study have shown that the ectopic expression of Wnt5a (a Fzd2 ligand) promotes OSCC cell migration and invasion in vitro (17), suggesting that the Wnt5a-Fzd2 pathway may be involved in OSCC metastasis. Although the overexpression of Fzd2 has been identified in a variety of OSCC cell lines (25), its functional role in OSCC development and progression remains elusive. The present study was thus conducted to examine the potential effects of Fzd2 on OSCC cell migration and invasion through loss- or gain-of-function experiments.

The most common location for OSCC is tongue (26), and therefore the TSCCa cell line established from tongue SCC (27) was used in the present study.Loss- or gain-of-function of Fzd2 was performed in TSCCa cells, respectively. The results of the present study showed that the migratory and invasive abilities of TSCCa cells were enhanced when the endogenous Fzd 2 was upregulated, but suppressed when Fzd2 was downregulated. These results suggested that Fzd2 played an oncogenic role in OSCC cells in vitro.

The metastatic process requires cancer cells to exit the primary tumor sites and to acquire migratory and invasive capabilities, in which EMT plays a critical role (20). EMT initiation leads to the destabilization of epithelial cell-cell junctions, which is accompanied by the degradation of multiple junction proteins (28). Since the downregulation of E-cadherin is the hallmark of EMT as previously described (19), we determined whether Fzd2 affected its expression in OSCC cells. We found that while Fzd2 overexpression induced a marked reduction in E-cadherin expression in TSCCa cells, Fzd2 knockdown induced an increase in E-cadherin. Opposite expression alterations in E-cadherin transcriptional suppressors, Snail and Slug (20), were observed in these cells. It has been reported that changes in the intermediate filament are also associated with cell motility and invasive abilities (20). Vimentin filaments usually replace intermediate cytokeratin filaments in mesenchymal-like cells that potentially favor local invasion and metastasis (29). Our results showed that TSCCa cells with enhanced migratory and invasive properties had a higher expression of vimentin, and that vimentin expression was positively regulated by Fzd2. In accordance with a comprehensive study conducted in liver, lung, colon and breast cancer cell lines (30), the present study demonstrated that Fzd2 played a role in the EMT process of OSCC cells at least partly through regulation of EMT-related factors.

ECM is a highly dynamic structure and its dysregulation has been recognized to contribute to numerous pathological conditions, including invasive cancers (22). Cancer cells use multiple modes to invade through ECM (31), and this process is mediated by specific enzymes that are responsible for ECM degradation such as metalloproteinases (22). MMP-2, -9 and -13 are major metalloproteinases, the increased expression levels of which are frequently observed in OSCCs (32-34). The present results showing that the expression levels of these MMPs in TSCCa cells were positively regulated by Fzd2 suggest that the MMPs were involved in Fzd2-mediated OSCC cell migration and invasion. ADAM proteins share a metalloproteinase domain with MMPs, and have been classified as membrane-anchored ADAMs and secreted ADAMs with thrombospondin motifs, referred to as ADAMTSs (23).
ADAMTS5 is a representative metalloproteinase in the ADAM family, and accumulated lines of evidence have shown that these matrix-degrading proteases are expressed in malignant tumors and participate in tumorigenesis (23). Notably, ADAMTS5 is markedly overexpressed in laryngeal SCC tissues as compared to normal tissues (35). The aberrant overexpression of ADAMTS5 may be associated with the tumorigenesis of OSCC. To the best of our knowledge, the present study is the first to identify that the expression of ADAMTS5 was regulated by Fzd2 in TSCCa cells.

Earlier studies have demonstrated that $\beta$-catenin-dependent (canonical) and $\beta$-catenin-independent (non-canonical) signaling can be activated by Fzd2 (36). Recently, the STAT3 signaling pathway has been identified as a new non-canonical pathway downstream of Fzd2 in cancer cell lines (30). Corresponding to such previous results, we also found that Fzd2 overexpression enhanced OSCC cell migration and invasion at least partly by promoting STAT3 signaling activation. Notably, it has been reported that the administration of recombinant Wnt5a promotes OSCC cell migration through the Wnt/planar cell polarity (PCP) pathway and/or the $\mathrm{Wnt} / \mathrm{Ca}^{2+}$ pathway (17). Given the fact that Wnt5a can be activated by Fzd2 in an autocrine-positive feedback manner (30), further studies are being conducted in our laboratory to investigate whether Fzd 2 mediates the malignant behaviors of OSCC cells through the $\mathrm{Wnt} / \mathrm{PCP}$ and/or $\mathrm{Wnt} / \mathrm{Ca}^{2+}$ pathway.

In summary, the present study provides evidence that Fzd2 mediates the migration and invasion of OSCC cells, at least partly by regulating the STAT3 signaling pathway. These results suggest Fzd2 is a novel therapeutic target for OSCC.

\section{Acknowledgements}

The present study was supported by grants from the Science and Technology Project of Shenyang City (no. F14-158-9-38), the Youth Startup Foundation of School of Stomatology, China Medical University (no. K101593-15-01) and the Department of Education, Liaoning Province (no.: L2015597).

\section{References}

1. Knopf A, Lempart J, Bas M, Slotta-Huspenina J, Mansour N and Fritsche MK: Oncogenes and tumor suppressor genes in squamous cell carcinoma of the tongue in young patients. Oncotarget 6: 3443-3451, 2015.

2. Taguchi T, Nishimura G, Takahashi M, Komatsu M, Sano D, Sakuma N, Arai Y, Yamashita Y, Shiono O, Hirama M, et al: Treatment results and prognostic factors for advanced squamous cell carcinoma of the larynx treated with concurrent chemoradiotherapy. Cancer Chemother Pharmacol 72: 837-843, 2013.

3. Zitelli KB,Zedek D, Ranganathan P and Amerson EH: Squamous cell carcinoma of the lip associated with adalimumab therapy for ankylosing spondylitis: A case report and review of TNF- $\alpha$ inhibitors and cutaneous carcinoma risk. Cutis 92: 35-39, 2013.

4. Dionne KR, Warnakulasuriya S, Zain RB and Cheong SC: Potentially malignant disorders of the oral cavity: Current practice and future directions in the clinic and laboratory. Int $\mathrm{J}$ Cancer 136: 503-515, 2015.

5. Wenig BM: Squamous cell carcinoma of the upper aerodigestive tract: Precursors and problematic variants. Mod Pathol 15: 229-254, 2002.

6. Han S, Chen Y, Ge X, Zhang M, Wang J, Zhao Q, He J and Wang Z: Epidemiology and cost analysis for patients with oral cancer in a university hospital in China. BMC Public Health 10: 196, 2010. 
7. Fury MG, Xiao H, Sherman EJ, Baxi S, Smith-Marrone S, Schupak K, Gewanter R, Gelblum D, Haque S, Schoder H, et al: A phase II trial of bevacizumab + cetuximab + cisplatin with concurrent intensity modulated radiation therapy (IMRT) for patients with stage III/IVB head and neck squamous cell carcinoma (HNSCC). Head Neck: Mar 17, 2015 (Epub ahead of print). doi: 10.1002/hed.24041.

8. Simpson DR, Mell LK and Cohen EE: Targeting the PI3K/AKT/ mTOR pathway in squamous cell carcinoma of the head and neck. Oral Oncol 51: 291-298, 2015.

9. Sagara N, Toda G, Hirai M, Terada M and Katoh M: Molecular cloning, differential expression, and chromosomal localization of human frizzled-1, frizzled-2, and frizzled-7. Biochem Biophys Res Commun 252: 117-122, 1998.

10. Peifer $M$ and Polakis P: Wnt signaling in oncogenesis and embryogenesis - a look outside the nucleus. Science 287: 1606-1609, 2000.

11. Ueno K, Hirata H, Hinoda Y and Dahiya R: Frizzled homolog proteins, microRNAs and Wnt signaling in cancer. Int J Cancer 132: 1731-1740, 2013

12. Lee HC, Kim M and Wands JR: Wnt/Frizzled signaling in hepatocellular carcinoma. Front Biosci 11: 1901-1915, 2006.

13. Hlubek F, Spaderna S, Schmalhofer O, Jung A, Kirchner T and Brabletz T: Wnt/FZD signaling and colorectal cancer morphogenesis. Front Biosci 12: 458-470, 2007.

14. Gurney A, Axelrod F, Bond CJ, Cain J, Chartier C, Donigan L, Fischer M, Chaudhari A, Ji M, Kapoun AM, et al: Wnt pathway inhibition via the targeting of Frizzled receptors results in decreased growth and tumorigenicity of human tumors. Proc Natl Acad Sci USA 109: 11717-11722, 2012.

15. Ding LC, Huang XY, Zheng FF, Xie J, She L, Feng Y, Su BH, Zheng DL and Lu YG: FZD2 inhibits the cell growth and migration of salivary adenoid cystic carcinomas. Oncol Rep: Feb 19, 2015 (Epub ahead of print). doi: 10.3892/or.2015.3811.

16. Rhee CS, Sen M, Lu D, Wu C, Leoni L, Rubin J, Corr M and Carson DA: Wnt and frizzled receptors as potential targets for immunotherapy in head and neck squamous cell carcinomas. Oncogene 21: 6598-6605, 2002.

17. Prgomet Z, Axelsson L, Lindberg P and Andersson T: Migration and invasion of oral squamous carcinoma cells is promoted by WNT5A, a regulator of cancer progression. J Oral Pathol Med: Dec 2, 2014 (Epub ahead of print). doi: 10.1111/jop.12292.

18. Schefe JH, Lehmann KE, Buschmann IR, Unger T and FunkeKaiser H: Quantitative real-time RT-PCR data analysis: Current concepts and the novel 'gene expression's CT difference' formula. J Mol Med Berl 84: 901-910, 2006.

19. De Craene B and Berx G: Regulatory networks defining EMT during cancer initiation and progression. Nat Rev Cancer 13: 97-110, 2013

20. Lamouille S, Xu J and Derynck R: Molecular mechanisms of epithelial-mesenchymal transition. Nat Rev Mol Cell Biol 15: 178-196, 2014

21. van Goor H, Melenhorst WB, Turner AJ and Holgate ST: Adamalysins in biology and disease. J Pathol 219: 277-286, 2009.

22. Bonnans C, Chou J and Werb Z: Remodelling the extracellular matrix in development and disease. Nat Rev Mol Cell Biol 15: 786-801, 2014.
23. Mochizuki S and Okada Y: ADAMs in cancer cell proliferation and progression. Cancer Sci 98: 621-628, 2007.

24. Yu H, Lee H, Herrmann A, Buettner R and Jove R: Revisiting STAT3 signalling in cancer: New and unexpected biological functions. Nat Rev Cancer 14: 736-746, 2014

25. Díaz Prado SM, Medina Villaamil V, Aparicio Gallego G, Blanco Calvo M, López Cedrún JL, Sironvalle Soliva S, Valladares Ayerbes M, García Campelo $R$ and Antón Aparicio LM: Expression of Wnt gene family and frizzled receptors in head and neck squamous cell carcinomas. Virchows Arch 455: 67-75, 2009.

26. Sano D and Myers JN: Metastasis of squamous cell carcinoma of the oral tongue. Cancer Metastasis Rev 26: 645-662, 2007.

27. Zhang YX, Yu SB, Ou-Yang JP, Xia D, Wang M and Li JR: Effect of protein kinase $\mathrm{C}$ alpha, caspase-3, and survivin on apoptosis of oral cancer cells induced by staurosporine. Acta Pharmacol Sin 26: 1365-1372, 2005.

28. Yilmaz $\mathrm{M}$ and Christofori G: EMT, the cytoskeleton, and cancer cell invasion. Cancer Metastasis Rev 28: 15-33, 2009.

29. Huang RY, Guilford P and Thiery JP: Early events in cell adhesion and polarity during epithelial-mesenchymal transition. J Cell Sci 125: 4417-4422, 2012.

30. Gujral TS, Chan M, Peshkin L, Sorger PK, Kirschner MW and MacBeath G: A noncanonical Frizzled2 pathway regulates epithelial-mesenchymal transition and metastasis. Cell 159 844-856, 2014.

31. Orgaz JL, Pandya P, Dalmeida R, Karagiannis P, SanchezLaorden B, Viros A, Albrengues J, Nestle FO, Ridley AJ, Gaggioli C, et al: Diverse matrix metalloproteinase functions regulate cancer amoeboid migration. Nat Commun 5: 4255, 2014.

32. Chiang WC, Wong YK, Lin SC, Chang KW and Liu CJ: Increase of MMP-13 expression in multi-stage oral carcinogenesis and epigallocatechin-3-gallate suppress MMP-13 expression. Oral Dis 12: 27-33, 2006.

33. Henriques AC, de Matos FR, Galvão HC and Freitas RA: Immunohistochemical expression of MMP-9 and VEGF in squamous cell carcinoma of the tongue. J Oral Sci 54: 105-111, 2012.

34. Ruokolainen H, Pääkkö P and Turpeenniemi-Hujanen T: Tissue and circulating immunoreactive protein for MMP-2 and TIMP-2 in head and neck squamous cell carcinoma - tissue immunoreactivity predicts aggressive clinical course. Mod Pathol 19: 208-217, 2006.

35. Filou S, Stylianou M, Triantaphyllidou IE, Papadas T, Mastronikolis NS, Goumas PD, Papachristou DJ, Ravazoula P, Skandalis SS and Vynios DH: Expression and distribution of aggrecanases in human larynx: ADAMTS-5/aggrecanase-2 is the main aggrecanase in laryngeal carcinoma. Biochimie 95: 725-734, 2013 .

36. Grumolato L, Liu G, Mong P, Mudbhary R, Biswas R, Arroyave R, Vijayakumar S, Economides AN and Aaronson SA: Canonical and noncanonical Wnts use a common mechanism to activate completely unrelated coreceptors. Genes Dev 24: 2517-2530, 2010 . 